\title{
Successful localization and treatment for ectopic adrenocorticotrophic hormone secretion in a rare case of possible Tx N2 MO carcinoid tumor with Cushing syndrome
}

\author{
Takafumi Sugawara, MD, ${ }^{a}$ Masami Sato, MD, ${ }^{a}$ Keiichi Itoi, MD, ${ }^{\text {b }}$ Akira Sugawara, MD, ${ }^{\mathrm{b}}$ Yasushi Matsuda, MD, ${ }^{a}$ \\ Kazuyoshi Shimada, MD, ${ }^{a}$ Tetsu Sado, MD, ${ }^{a}$ Shulin Wu, MD, ${ }^{a}$ and Takashi Kondo, MD, ${ }^{a}$ Sendai, Japan
}

$\mathrm{I}$ $\mathrm{t}$ is widely known that a carcinoid tumor can produce various hormones and is sometimes detected on the basis of the presence of various symptoms caused by the hormones. Cushing syndrome is widely known as one of these symptoms. Many cases of Cushing syndrome have been reported, but occasionally, it is very difficult to detect a tumor that produces adrenocorticotrophic hormone (ACTH). ${ }^{1,2}$ We herein report a rare case of a carcinoid tumor that metastasized to a lymph node, resulting in production of ACTH. Although the lymph node was not enlarged by tumor metastasis, we were able to localize the lymph nodes as the origin of ectopic ACTH production by means of ${ }^{111} \mathrm{In}$-octreotide scanning. The lymph nodes were completely resected with thoracoscopic surgery, and the symptoms successfully disappeared after the operation.

\section{Clinical Summary}

The patient was a 55-year-old woman who had had hypertension since 1991. However, she refused any treatments. In 1996, she reported having facial edema. Hypertension and hypokalemia were detected during examination. Both plasma ACTH and serum cortisol levels were increased (ACTH, $121 \mathrm{pg} / \mathrm{mL}$ [normal range, 4.4-48.0 pg/mL]; cortisol, $26.6 \mu \mathrm{g} / \mathrm{dL}$ [normal range, 1.39-24.20 $\mathrm{g} / \mathrm{dL}]$ ). Increased 17-hydroxycorticosteroids and 17-ketosteroids in the urine were also noted. She was given a diagnosis of Cushing syndrome. Brain magnetic resonance imaging and abdominal computed tomography (CT) failed to detect any tumor mass. The administration of an oral antihypertensive agent was started, and the serum cortisol level decreased to the normal value, although the plasma ACTH level was still increased $(95.4 \mathrm{pg} / \mathrm{mL})$. Wholebody screening was performed, and an abnormal mass was detected by means of chest CT in February 1996 (Figure 1, A), suggesting lymphadenopathy in the anterior mediastinum. No change in tumor size was seen with serial examination until 2001.

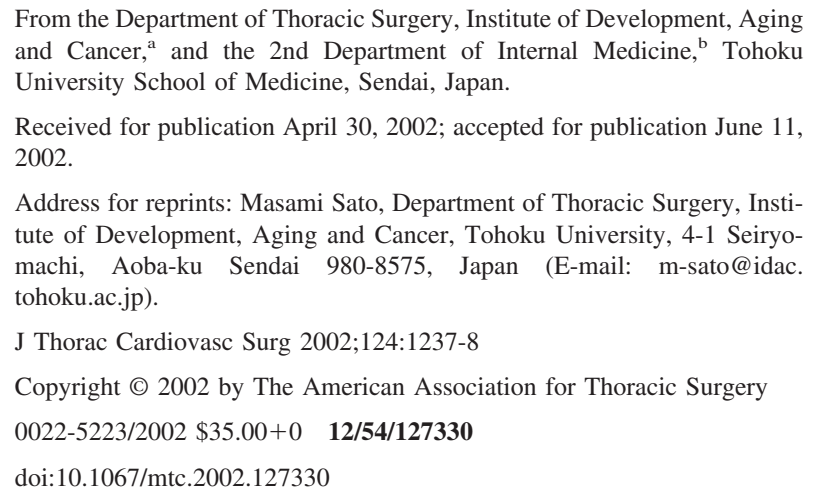

Thus, we were not able to obtain proof that this mediastinal mass was the origin of ectopic ACTH secretion. In 2001, an ${ }^{111}$ Inoctreotide scan was performed, which showed a hot spot in the right upper mediastinum (Figure $1, B$ ). ${ }^{3}$ The ACTH value in the superior vena cava ${ }^{4}$ was also shown to be higher than that in the peripheral veins. All these findings strongly suggested that the mediastinal mass was an ACTH-producing tumor.

The patient underwent tumor resection with a thoracoscope on May 18, 2001. The tumor seemed to be a solitary, enlarged lymph node, and no other tumor was detected in either the right lung or the mediastinum by means of thoracoscopy. Pathologic examination showed the tumor to be a pair of metastatic lymph nodes (No. 3 [1/6] and No. 4 [4/15]) involved with an atypical carcinoid tumor (Figure 2). Immunohistochemical staining results for ACTH was positive. Examination with a fiberoptic scope targeting the gastrointestinal region, the colon, and the bronchi after the operation failed to find the primary region of the tumor. Plasma ACTH and serum cortisol values decreased to normal levels after the operation.

\section{Discussion}

Cushing syndrome related to ectopic ACTH production is associated with a variety of solid tumors (ie, lung cancer, bronchial carcinoid, thymic carcinoid, and gastrointestinal pheochromocytoma). On occasion, the primary lesion of the tumor releasing ACTH cannot be detected. In our case a tumor was detected by means of $\mathrm{CT}$ and was confirmed to be an ACTH-producing tumor on the basis of the scintigraphic findings. Pathologic diagnosis was lymph node metastasis of a carcinoid; a primary lesion, however, could not be identified despite thorough examinations. Aniszewski and colleagues ${ }^{5}$ reported that no tumor was detected in $17(16 \%)$ of 106 cases of ectopic ACTH syndrome. They found bronchial carcinoid to be the most frequent cause of this paraneoplastic disorder. Several reports have also shown the frequent involvement of regional lymph nodes in cases of atypical carcinoid tumor. Our patient had mediastinal nodal involvement but no detectable primary lesion in either the lung or the thymus. After removal, symptoms associated with Cushing syndrome disappeared, and the value of the ACTH level returned to normal. Thus, it is suggested that the lymph nodes removed by means of thoracoscopic surgery were the major origin of ectopic ACTH in our case. There have been some reports of Tx N2 M0 lung cancer. Thus, we concluded that this case was a carcinoid tumor with Tx N2 M0 stage IIIA disease. However, we could not find any reports of Tx N2 M0 carcinoid tumors. Careful follow-up for detection of a possible occult primary pulmonary lesion is required. 

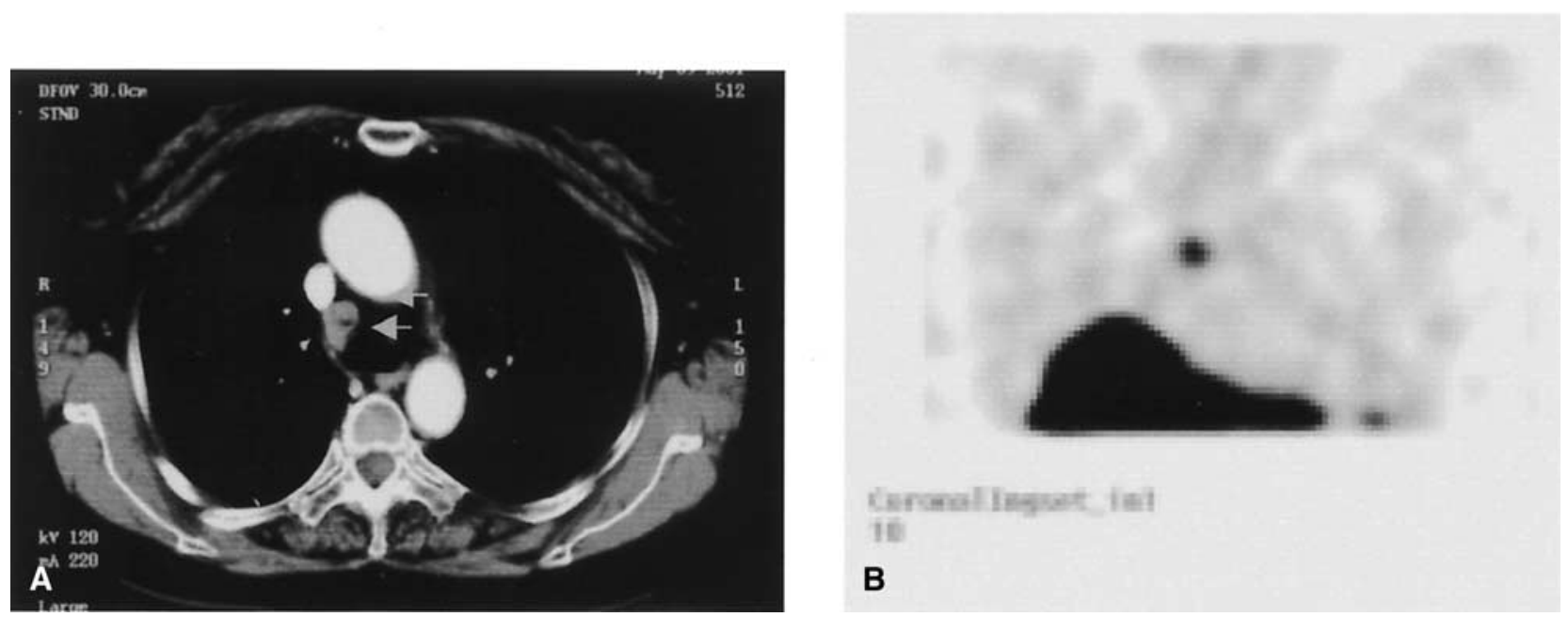

Figure 1. A, Chest CT showed an abnormal mass (arrows) in the right upper mediastinum. B, Octoreoscan revealed a hot spot in the mediastinum. The liver also showed a hot spot after uptake of radiolabeled material.


Figure 2. A, Low-power view of lymph nodes prepared for histologic examination. B, There were many cells of palisade structure infiltrating among lymphocytes. (Original magnification 10X, hematoxylin and eosin.)

\section{References}

1. Jex RK, van Heerden JA, Carpenter PC, Grant CS. Ectopic ACTH syndrome. Diagnostic and therapeutic aspects. Am J Surg. 1985;149: 276-82.

2. Shrager JB, Wright CD, Wain JC, Torchiana DF, Grillo HC, Mathisen DJ. Bronchopulmonary carcinoid tumors associated with Cushing's syndrome: a more aggressive variant of typical carcinoid. J Thorac Cardiovasc Surg. 1997;114:367-75.

3. Mansi L, Rambaldi PF, Panza N, Esposito D, Esposito V, Pastore V.
Diagnosis and radioguided surgery with ${ }^{111}$ In-pentetreotide in a patient with paraneoplastic Cushing's syndrome due to a bronchial carcinoid. Eur J Endocrinol. 1997;137:688-90.

4. Oldfield EH, Doppman JL, Nieman LK, Chrousos GP, Miller DL, Katz DA, et al. Petrosal sinus sampling with and without corticotropin-releasing hormone for the differential diagnosis of Cushing's syndrome. N Engl J Med. 1991;325:897-905.

5. Aniszewski JP, Young WF Jr, Thompson GB, Grant CS, van Heerden JA. Cushing syndrome due to ectopic adrenocorticotropic hormone secretion. World J Surg. 2001;25:934-40. 